\title{
Behindert Sponsoring den Erkenntnisprozess in der Medizin?
}

\author{
For and Against: Does Sponsoring Impede the Growth of Knowledge \\ in Medicine?
}

Bibliografie

DOI $10.1055 / \mathrm{s}-2006-951952$

Psychiat Prax 2007; 34:

162-164 @ Georg Thieme

Verlag KG Stuttgart · New York . ISSN 0303-4259

Korrespondenzadressen

Prof. Dr. Asmus Finzen

Spalenvorstadt 3

4051 Basel, Schweiz

asmus.finzen@vtxmail.ch

Prof. Dr. med. Jürgen Fritze

Verband der privaten Krankenversicherung e.V.

Bayenthalgürtel 26

50968 Köln

juergen.fritze@dgn.de

\section{Pro} Sponsoring reden, meinen wir Industrie - nicht Daimler-Chrysler, die deutsche Bank, Versace oder Aldi. Wir meinen die Förderung medizinischer Forschung und Fortbildung durch einen medizinisch-industriellen Komplex, der weltweit jährlich mehr als 500 Milliarden Dollar umsetzt und der ein massives Interesse daran hat, dass wir als Gate Keeper der Arzneiverordnung ihm wohlwollen. Und die meisten von uns wollen ihm wohl, wir dankbaren Ärzte, die wir seit unserer Ausbildung unsere Freude an den kleinen und großen Gaben der Arzneimittelhersteller haben [1].

Die Kugelschreiber auf unserem Schreibtisch, unsere Terminkalender, ein Teil unserer Fachbücher sind solche Geschenke - wenn wir wichtig sind, gelegentlich sogar ein Laptop. Die meisten Fortbildungsveranstaltungen, die wir besuchen, sind industriegesponsert. Und wenn wir Vorträge halten, nehmen wir (ich auch) gern - ordentliche Honorare entgegen, von denen wir wissen, dass sie gesponsert sind.

Wir sind nicht bestechlich, natürlich nicht - dazu braucht es mehr als einen Kugelschreiber. Aber wenn wir ehrlich mit uns sind, wissen wir, dass es nichts umsonst gibt. Es ist schlicht ein Gebot der Höflichkeit, dass wir uns als Referenten nicht allzu kritisch über das Präparat des Hauptsponsors äußern, das diesen erst zum Sponsoring motiviert. Und es ist nur menschlich, dass wir dem sympathischen Repräsentanten dieses Sponsors, mit dem wir einen netten Abend verbracht haben, bei seinem nächsten Besuch aufmerksamer zu hören als einem beliebigen anderen. „Ärzte sind wie andre Menschen", schreibt Bernhard Shaw in seiner Vorrede über die Ärzte vor ziemlich genau 100 Jahren [2]; und daran hat sich nichts geändert.

Warum soll es nicht möglich sein, dass die einkommensstarken Mitglieder eines Zweigs des
Gesundheitswesens, der allein in unserem Land mehrere 100 Milliarden Euro umsetzt, ihre Fortbildung aus eigener Kraft finanzieren? Ich bin nicht weltfremd. Ich weiß, dass wir uns seit 50 Jahren zunehmend auf die Fremdfinanzierung durch die Industrie eingelassen haben, und dass wir nicht rechtzeitig gegengesteuert haben. Wir haben uns auf dem Gebiet der Fortbildung unseren Sponsoren mehr oder weniger ausgeliefert. Wir sind auf ihren guten Willen angewiesen; und im Zeichen knapper Kassen ist es nur schwer möglich, die öffentlichen Träger des Gesundheitswesens davon zu überzeugen, den Stab zu übernehmen. Wir müssten also in unsere eigene Tasche greifen. Aber da wir nicht bestechlich sind, sehen wir nicht ein, warum wir das tun sollen. Also bleibt vorerst alles beim Alten.

Schließlich bietet auch unsere Fachgesellschaft fast nur obligatorische Fortbildungsveranstaltungen an, die industriegesponsert sind. Schließlich finanzieren auch unsere Standesorganisationen ihr offizielles Mitteilungsblatt über Anzeigen der Industrie und, wie man hört, aus den Überschüssen sich selber, statt einen Beitrag zu einer werbefreien unabhängigen medizinischen Zeitschrift, wie etwa dem British Medical Journal, zu leisten. $\mathrm{Zu}$ glauben, dass das allgegenwärtige Sponsoring zu einer unabhängigen Meinungsbildung von uns Ärzten beiträgt, ist Traumtänzerei. Aber jede Beeinträchtigung dieser Unabhängigkeit behindert zwangsläufig den medizinischen Fortschritt: Gesponsert wird immer aus dem Budget jener Medikamente, die die Sponsoren aktiv bewerben, auch wenn die Themen der gesponserten Veranstaltungen damit primär nichts zu tun zu haben scheinen.

Jürgen Fritze ist in seiner Verteidigung des Sponsoring ausschließlich auf die klinische Forschung eingegangen, genauer auf die klinische Prüfung von Medikamenten. Diese ist notwendig. Und Fritze fordert mit Recht, dass dafür zahlen soll, wer den größten Vorteil davon hat. Aber ist das 
Sponsoring? Ist die klinische Prüfung von neuen Arzneimitteln nicht vielmehr Auftragsforschung? Sollte die Mitwirkung daran nicht durch Werkverträge geregelt werden, die neben den wissenschaftlichen Rahmenbedingungen die Erstattung der Vollkosten an die prüfenden Institutionen und angemessene Entschädigungen für die mitwirkenden Probanden enthalten müssen?

Es steht außer Frage, dass die Arzneimittelforschung - wenn sie denn innovativ ist - den medizinischen Fortschritt fördert. Das Problem liegt auf einer anderen Ebene. Weil es um so viel Geld geht, dient solche Forschung allzu häufig vorrangig dem Ziel, den Patentschutz zu verlängern, ohne dass die Kranken davon profitieren, z.B. durch zweifelhafte Indikationserweiterungen auf Kinder. Zugleich stellen die Arzneimittelhersteller fast immer jede Forschung und die Förderung bewährter Medikamente ein - einschließlich des damit verknüpften Sponsoring - , sobald die ersten Generika auf den Markt kommen. Das hat gelegentlich dramatische Folgen für die Förderung von Fortbildungsmaßnahmen, etwa wenn sich ein Produzent als Konsequenz aus einem Fachgebiet, etwa der Psychiatrie, zurückzieht.

Aus der Sicht des Arzneimittelherstellers ist das nur legitim. Aber es behindert den medizinischen Fortschritt, wenn Stund an die Fortbildung über solche bewährten Substanzen nicht mehr finanzierbar ist und entsprechend nicht mehr stattfindet - noch mehr allerdings, wenn die Förderungsmittel von da an mit der Werbung für pseudoinnovative Homologe verknüpft werden, etwa gereinigte Razemate oder methysierte desmethylisierte Substanzen, die dann als besonders wirksam verkauft werden. Das wiederum behindert den medizinischen Fortschritt. Wer mehr wissen will, möge bei Marcia Angell [3] nachlesen, der langjährigen Herausgeberin des New England Journal of Medicine: „The Truth about Drug Companies - How they deceive us, and what to do about it". Sie stellt in ihrem bitterbösen Buch neben vielem anderen vor allem auch die fortschrittsbehindernden Aspekte von industriegeförderter Fortbildung und Forschung an den Pranger: Marketing masquerading as Education und Marketing masquerading as Research. Dem ist nichts hinzuzufügen.

Die Abhängigkeit der Medizin von ihren Sponsoren ist ein Dilemma. Es kann nicht darum gehen, es hier und jetzt aufzulösen. Als erstes muss uns bewusst werden, dass es eins ist. Wir können getrost unterstellen, dass es unseren Sponsoren ähnlich wie uns darum geht, kranken Menschen auf die bestmögliche Weise zu helfen. Aber jenseits dieses gemeinsamen Anliegens, das uns verbindet, trennen uns widerstreitende Interessen. Das ist von der Natur der Sache her unvermeidbar. Denn die Medizin ist zugleich ein Markt, auf dem es weltweit um Billionen geht. $\mathrm{Zu}$ glauben, dass man dessen Versuchungen durch Verhaltenskodizes regeln kann, ist mehr als naiv. Das Ziel der Gewinnmaximierung konkurriert dort zwangsläufig mit dem Ziel des medizinischen Fortschritts und, was wichtiger ist, mit dem Patientenwohl.

\section{Kontra}

Es besteht kein Zweifel, dass die Herkunft der Finanzierung von Studien Form und Inhalt einer Publikation oder Präsentation beeinflussen kann [4], auch in der Psychopharmakologie [5]. Einflüsse können auch mittelbar und auch ungewollt erfolgen, indem das Studiendesign ein bestimmtes Produkt begünstigt oder „ungünstige“ Studienergebnisse nicht zur Publikation eingereicht oder angenommen (publication bias) werden [6]. Das ist auch für die $\mathrm{Zu}$ kunft zu erwarten.

Um die Aufmerksamkeit für potenziell verzerrte Informationen zu schärfen, sind mögliche Interessenkonflikte darzulegen. Die Wirksamkeit dieses Verfahrens ist bezweifelt und stringente Regelungen sind gefordert worden [7]. Um zumindest der publication bias vorzubeugen, ist die verpflichtende Registrierung in öffentlich zugänglichen Studienregistern vorgesehen, in Europa aber noch nicht umgesetzt.

Konventionell wird der Finanzier einer Studie als Sponsor bezeichnet. Das Bundesfinanzministerium (BMF-Schreiben vom 18.2.1998) hat aber Sponsoring definiert als „die Gewährung von Geld oder geldwerten Vorteilen durch Unternehmen zur Förderung von Personen, Gruppen und/oder Organisationen in sportlichen, kulturellen, kirchlichen, wissenschaftlichen, sozialen, ökologischen oder ähnlich bedeutsamen gesellschaftspolitischen Bereichen, mit der regelmäßig auch eigene unternehmensbezogene Ziele der Werbung oder Öffentlichkeitsarbeit verfolgt werden“. Dem Sponsoring im Sinne dieser Legaldefinition steht also keine geldwerte, angemessene Gegenleistung des Empfängers der Sponsorship gegenüber. Solches Sponsoring anzunehmen, ist dem Arzt gemäß Berufsordnung ( $\$ 32 \mathrm{MBO}$ ) grundsätzlich verboten, weil „hierdurch der Eindruck erweckt wird, dass die Unabhängigkeit der ärztlichen Entscheidung beeinflusst wird“.

Es dürfte aber kaum streitig sein, dass einem Arzt und einer ärztlich geleiteten Einrichtung für erbrachte Leistungen eine Vergütung zusteht. Das leuchtet spätestens ein, wenn man bedenkt, dass Arzt und Einrichtung in der „geopferten“ Zeit anderweitig hätten Einnahmen erzielen können. Das ist auch ansonsten selbstverständlich im Geschäftsverkehr. Das muss also auch für Leistungen gelten, die im Auftrag von Unternehmen der pharmazeutischen oder Medizinprodukteindustrie erbracht werden. Wird der Begriff Sponsoring im allgemeineren Sinne der Vergütung (funding) verwendet, dann hat die Höhe dieser Vergütung gemäß §33 MBO in angemessenem Verhältnis zur ärztlich-wissenschaftlichen Leistung zu stehen. Angemessen bedeutet - z. B. in Begriffen der Gebührenordnung ( $§ \S 5$ und 6 GOÄ) - den Kosten, der Schwierigkeit und dem Zeitaufwand der Leistung sowie den Umständen bei der Ausführung entsprechend. Was dies im Detail bedeutet, kann hier außer acht bleiben.

Die Leistungen müssen ihrer Art nach mit dem ärztlichen Ethos und dem darauf aufbauenden Berufsrecht vereinbar sein. Die MBO (§33) nennt exemplarisch die „Entwicklung, Erprobung und Begutachtung “ von Arznei-, Heil- und Hilfsmitteln oder Medizinprodukten und verbietet ( $\$ 34$ ) explizit, „über Arznei-, Heilund Hilfsmittel, Körperpflegemittel oder ähnliche Waren Werbevorträge zu halten oder zur Werbung bestimmte Gutachten zu erstellen“. Gemäß $§ 15$ MBO sind „in Publikationen von Forschungsergebnissen die Beziehungen der Ärztin oder des Arztes zum Auftraggeber und dessen Interessen offenzulegen“ (was implizit auch für Vorträge gilt). Zum Sponsoring von Fortbildungsveranstaltungen fordert $\S 35 \mathrm{MBO}$, Beziehungen zum Sponsor seien bei der Ankündigung und Durchführung offen darzulegen. 
Den Zulassungsbehörden sind alle Studien anzuzeigen. Bei zulassungsrelevanten Studien haben die Zulassungsbehörden $\mathrm{Zu}$ griff auf die kompletten Studienunterlagen, also auch die unternehmensinternen, vertraulichen Studienprotokolle und Studienberichte bis hin zu den Rohdaten. Die Zulassungsbehörden sind damit nicht den Risiken der publication bias ausgeliefert. Die formale und inhaltliche Qualität der den Zulassungsbehörden zugänglichen Informationen übersteigt damit grundsätzlich die Qualität von Publikationen. Methodische Schwächen (z.B. Bias im Design) der Studien zugunsten eines Produktes werden also in der Zulassungsentscheidung gewürdigt.

Wenn sich Metaanalysen, auch auf Basis systematischer Literaturrecherche, ausschließlich auf publizierte Daten stützen, besteht im Vergleich zu den Zulassungsbehörden ein erhöhtes Risiko verzerrter Information. Wirklich bedeutsam wird das Problem also verfälschter Erkenntnis (bias) bei Studien ohne Zulassungsrelevanz. Das sind insbesondere Studien zum Vergleich verschiedener Produkte, solange nur der Nachweis der Plazeboüberlegenheit Zulassungsvoraussetzung ist und nicht der Nachweis gleicher (Non-Inferiorität) oder gar überlegener Wirksamkeit. Ob diese Beschränkung hinreichend rational ist, spielt hier keine Rolle.

Was wären Alternativen zum Sponsoring im Sinne der angemessenen Vergütung erbrachter Leistungen (funding)? Leicht ist es, öffentliche Studienfinanzierung zu fordern. Schwer aber ist es, die notwendigen Ressourcen zu aquirieren. Würde die öffentliche Finanzierung aber tatsächlich vor verzerrter (bias) Präsentation von Daten schützen? Kaum, denn auch die Forscher selbst verfolgen Interessen (ohne Interesse findet Forschung nicht statt). Der Einfluss dieser Interessen scheint bisher nicht systematisch untersucht worden zu sein. Immerhin gibt es aber sogar gezielten Wissenschaftsbetrug [8], wozu wiederum systematische Untersuchungen weitgehend fehlen [9].

Öffentliche Finanzierung kann also verzerrte Information nicht ausschließen, allenfalls abmildern. Dennoch sind öffentlich finanzierte Studien selbstverständlich unbedingt zu fordern, insbesondere für Fragestellungen, für die es an - letztlich ökonomisch motivierten - Interessenten fehlt. Dies gilt zum Beispiel nahezu regelhaft für Produkte, deren Patentschutz ausgelaufen ist.

Letztlich ist es unausweichlich, dass derjenige, der von einer Forschung - ökonomisch - profitiert, diese Forschung auch zu finanzieren hat. Staatliche und andere gemeinnützige (auch durch Kostenträger) Forschungsförderung kann und darf Forschung grundsätzlich nur finanzieren, wenn keine Partikularinteressen den Forschungsgegenstand dominieren, das gesamtgesellschaftliche Interesse also im Vordergrund steht.

Zur Abwehr verfälschter Information in Forschung und Werbung hat sich der Verband forschender Arzneimittelhersteller (VFA) einen Verhaltenskodex gegeben [10], dessen Verletzung mit empfindlichen Sanktionen bedroht ist. Wirksamster Schutz gegen verfälschte Erkenntnis und Betrug ist und bleibt am Ende die mit den methodischen Richtlinien (z. B. GCP) der Zulassungsbehörden (z.B. Notes for Guidance der EMEA) konforme (und überwachte) Planung, Durchführung und Auswertung der Studien, die systematische Begutachtung der systematisiert zu erstellenden [11,12] Manuskripte - und die Kritikfähigkeit der Leser (und Zuhörer).

\section{Literatur}

1 Finzen A. Wir dankbaren Ärzte. Dtsch Ärztebl 2002; 99: A 766 - 769

2 Shaw GB. Des Doktors Dilemma. Frankfurt: Suhrkamp, 1991

3 Angell M. The Truth about the Drug Companies. NY: Random House, 2004

4 Lexchin J, Bero LA, Djulbegovic B, Clark O. Pharmaceutical industry sponsorship and research outcome and quality: systematic review. BMJ 2003; 326 (7400): 1167-1170

5 Heres S, Davis J, Maino K, Jetzinger E, Kissling W, Leucht S. Why olanzapine beats risperidone, risperidone beats quetiapine, and quetiapine beats olanzapine: an exploratory analysis of head-to-head comparison studies of second-generation antipsychotics. Am J Psychiatry 2006; 163 (2): $185-194$

6 Chan AW, Hrobjartsson A, Haahr MT, Gotzsche PC, Altman DG. Empirical evidence for selective reporting of outcomes in randomized trials: comparison of protocols to published articles. JAMA 2004; 291 (20): 2457-2465

7 Brennan TA, Rothman DJ, Blank L, Blumenthal D, Chimonas SC, Cohen JJ, Goldman J, Kassirer JP, Kimball H, Naughton J, Smelser N. Health industry practices that create conflicts of interest: a policy proposal for academic medical centers. JAMA 2006; 295 (4): 429- 433

8 Fenning TM. Fraud offers big rewards for relatively little risk. Nature 2004; 427 (6973): 393

9 Office of Research Integrity, Office of Public Health and Science, Department of Health and Human Services. Scientific Misconduct Investigations 1993 - 1997, 1998. http://ori.dhhs.gov/documents/misconduct_ investigations_1993_1997.pdf

10 Verein „Freiwillige Selbstkontrolle für die Arzneimittelindustrie e.V.“ FS Arzneimittelindustrie-Kodex. http://www.vfa.de/de/vfa/fs-arzneimit telindustrie/fsam-dokumente/fsa-kodex.html

11 Moher D, Cook DJ, Eastwood S, Olkin I, Rennie D, Stroup DF. Improving the quality of reports of meta-analyses of randomised controlled trials: the QUOROM statement. Quality of Reporting of Meta-analyses. Lancet 1999; 354 (9193): 1896-1900

12 Moher D, Schulz KF, Altman D CONSORT Group. The CONSORT Statement: revised recommendations for improving the quality of reports of parallel-group randomized trials 2001. Explore (NY) 2005; 1 (1): $40-45$ 